\title{
Study of Metamaterials and Analysis of Split Ring Resonators to Design Multiband and UWB Antennas
}

\author{
K. V. Ajetrao ${ }^{1}$ and A. P. Dhande ${ }^{2}$ \\ ${ }^{1}$ D Y Patil Institute of Engineering and Technology/Electronics and Telecommunication Department, Pimpri ,Pune, India \\ Email: kiran_ajetrao@rediffmail.com \\ ${ }^{2}$ Pune Institute of Computer Technology/Electronics and Telecommunication Department, Pune, India \\ Email: apdhande@ pict.edu
}

\begin{abstract}
Metamaterials are artificial structures which provide engineerable permeability and permeability. This paper gives classification of metamaterial based on available literature. In this paper Split Ring Resonator is used to explain the concept of metamaterials. Further the work is extended to design printed monopole antenna and effect of SRR to generate multiband antenna in the frequency band of $1 \mathrm{GHz}$ to $10 \mathrm{GHz}$. In next section of paper multiband antenna in converted to UWB antenna by modifying the ground structure of multiband antenna using SRR. Now day's multiband antenna with compact size is growing field of research and metamaterials promise small size, high efficient multiband and UWB antennas.
\end{abstract}

Index Terms - Electromagnetic Metamaterials, Monopole antenna, Multiband antenna, Split Ring Resonator.

\section{INTRODUCTION}

Electromagnetic metamaterials (MTMs) are broadly defined as artificial effectively homogeneous electromagnetic structures with unusual properties not readily available in nature.[1][3]. The concept of artificial materials first introduced by Jagadis Chandra Bose while conducting microwave experiments on twisted structures, these artificial structures are termed as chiral elements by todays terminology. In 1914 Lindman worked on artificial chiral media. In 1948 Kock worked on lightweight microwave lenses by arranging conducting spheres, disks, and strips periodically and effectively tailoring effective refractive index of the artificial media.[2] In 1967, Veselago theoretically proved plane wave propagation in material whose permittivity and permeability both were simultaneously negative.[4] Metamaterials shows negative permittivity and permeability. Many researchers are presently working on metamaterials and they have proposed various terminologies to metamaterials such as Left handed materials, negative refractive index materials, backward wave media(BW media), double negative metamaterials. Many research groups all over the world are now studying various aspects of this class of metamaterials, and several ideas and suggestions for future applications of these materials have been proposed.[2]

In this research paper review on metamaterial is given with some practical antenna designs in HFSS and its simulated results. In review of metamaterials author studied many paper and classified them according to the techniques used for generation of metamaterial for the design of antennas. Antennas are simulated by

Grenze ID: 01.GIJET.2.2.19

(C) Grenze Scientific Society, 2016 
considering FR4 substrate of dielectric constant of 4.4, thickness of $1.6 \mathrm{~mm}$ and loss tangent of 0.02 . Effect of SRR on monopole antenna design is studied and from the simulation results it is found that Multiband antennas of desired frequency can be achieved. Further the work is extended to analyse the result of CSRR and SRR to generate Ultra Wide Band antenna and is simulated and presented in this paper.

\section{Metamaterials And Its Classifications}

The Metamaterials are artificial structures which produce electromagnetic properties that are not found or difficult to obtain in nature. Metametrials are able to provide engineerable permittivity, permeability and index of refraction. To determine propagation of waves in matter, dielectric constant and magnetic permeability are the fundamental quantities.[1][2][3] Refractive index of substance is given by(1). [4]

$$
n^{2}=\varepsilon \mu
$$

Where $n, \mu$ and $\varepsilon$ are refractive index, permeability and permittivity of material respectively. These are real numbers if losses are not considered. Hence simultaneous change in sign of $\mu$ and $\varepsilon$ has no effect on properties of substance. If $\mu$ and $\varepsilon$ both are negative it contradicts fundamental laws of nature and therefore no material with $\mu$ and $\varepsilon$ values less than zero can exist. Fig. 1. shows classification materials based four possible combinations of sign of $\mu$ and $\varepsilon$ values. [1].

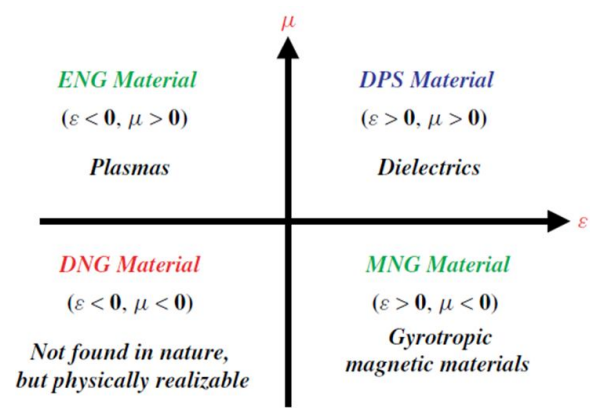

Figure. 1. Metamaterial classification based on $\varepsilon-\mu[1]$

I quadrant shows both $\mu$ and $\varepsilon$ are positive i.e. (DPS) double positive mediums do occur in nature. II quadrant is Epsilon negative (ENG) and IV quadrant is mu negative (MNG) materials. This concept is used to design metamaterial based antennas. III quadrant shows material with both $\mu$ and $\varepsilon$ are negative called as (DNG) double negative which are not available in nature but can be physically realized.[7] We use metamaterial antenna as it has benefits of small physical size, low cost, broad bandwidth, and good efficiency.[1][2][8] Metamaterials received great attention in research areas of physics and engineering. Microwave and milimiter wave applications of metamaterials are as couplers, resonators, negative refractive index lenses, small antennas, leaky wave antennas and absorbers.[9] Some interesting application of metamaterials is cloaking and invisible metamaterials. Here we will discuss some of its application in multiband and UWB antennas. Antennas which work in more than one frequency band for transmitting and receiving electromagnetic waves are called as multiband antennas. Multiband antennas are complex in design, structure and operations as compared to single band antenna [7][10] This paper reviews the development of metamaterial based antenna and are classified in the following categories.[7][10]

a. Composite Right Left Handed Transmission Line (CRLH-TL) based or dispersion engineered resonant multiband antennas which includes the antennas with negative-order modes and zerothorder resonators.

b. Multiband antennas based on the metamaterial loadings

c. Multiband and UWB antennas using Split Ring Resonators.

d. Multiband antennas loaded with metasurfaces.

\section{A. Concept of Split Ring Resonator}

An SRR shown in Fig. 2 is a metamaterial structure that produces both electric and magnetic responses. Split Ring resonator can be of any shape circular, square, triangular etc. An SRR element is the electromagnetic analog of an $L C$ circuit, in which the ring acts as an inductor and the gap as a capacitor. When the axis of the ring is parallel to the $H$-field, the $H$-field generates a current in the SRR, which gives 


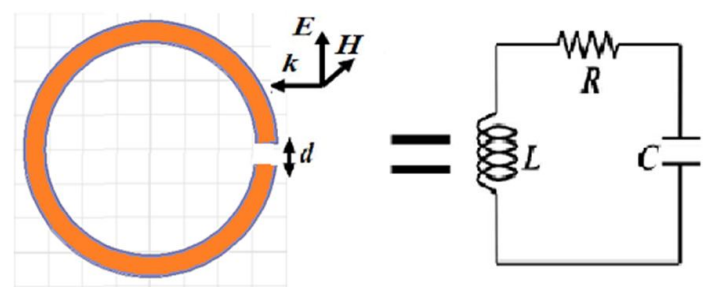

Fig 2 Split Ring Resonator

rise to a strong magnetic dipole and, hence, a magnetic resonance. If the gap of the SRR is parallel to the $E$-field, the $E$-field generates voltage variation at the gap, leading to a strong electric dipole, and this gives rise to an electric resonance. The modified SRR with both electric and magnetic resonances at two closely spaced frequencies is used to improve the bandwidth of the antenna. [11]

\section{B. Concept of Negative Effective Permeability in Microwave band}

According to Drudes relation (1) metals at optical frequencies are characterized by an electric permittivity that varies with frequency.[3]

Where

$$
\varepsilon(\omega)=\varepsilon_{0}\left(1-\frac{\omega_{p}^{2}}{\omega(\omega+i \gamma)}\right)
$$

$\omega_{p}^{2}=\frac{N_{c}^{2}}{m \varepsilon_{0}}$ is the plasma frequency. i.e. frequency with which the collection of free electrons oscillates in the presence of an external driving field. $\mathrm{N}$, e, and $\mathrm{m}$ are electron density, charge and mass respectively. $\gamma$ is the rate at which the amplitude of plasma oscillation decreases.

From equation (2) it is clear that when $\gamma=0$ and $\omega<\omega_{\mathrm{p}}$ value of $\varepsilon$ will be less than zero, it means the medium is characterized by a negative permittivity.[3]

\section{Monopole Antenna Design}

A general purpose dipole antenna (long thin wire antenna) with height $h 1=h 2=L / 2$, where $L$ is the total length of the antenna is shown in Fig. 3. [5]

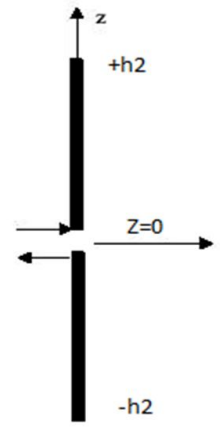

Fig 3. Dipole antenna total length of $h 1+h 2$

Two thin linear elements are used to construct dipole antenna. Two thin linear symmetrical elements of dipole antenna are fed at the centre by a balanced two wire transmission line. The antennas may be of any length, but it is assumed that the current distribution is sinusoidal. Current-distribution measurements indicate that this is a good assumption provided that the antenna is thin: That is, when the conductor diameter is less than $\lambda / 100$.

The current of the centre fed antenna of length $L$ at any point $z$ on the antenna is

$$
I(z)=I_{0} \sin \left[\frac{2 \pi}{\lambda}\left(\frac{L}{2}-|z|\right)\right]
$$


From dipole antenna concept monopole antenna is designed. For monopole antenna single ended sources can be used without baluns. $\lambda / 4$ monopole antenna display the same radiation pattern in the region above the ground as a half-wave dipole in free space as shown in Fig. 4.a/b. Monopole radiates only half as much power as the dipole.[6]

\section{A. Printed T Type monopole}

Based on the same concept, in this paper printed monopole antenna is designed and simulated using HFSS simulation tool. In simulation FR4 substrate with loss tangent of 0.02 , thickness of $1.6 \mathrm{~mm}$ and dielectric constant of 4.4 in considered.

Based on the simulation in HFSS monopole antenna is tuned to $2.4 \mathrm{GHz}$ frequency with return loss of 12.73dB. The frequency verses return loss response is as shown in Fig. 5.

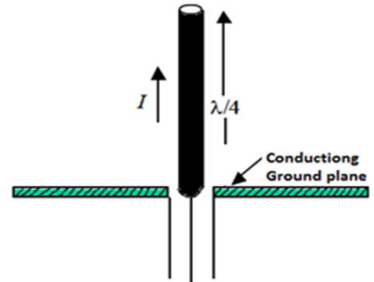

(a)

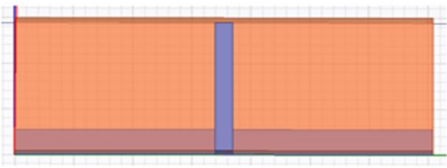

(b)

Figure 4. $\lambda / 4$ monopole antenna (a) Wired monopole antenna (b) Printed Monopole antenna in HFSS

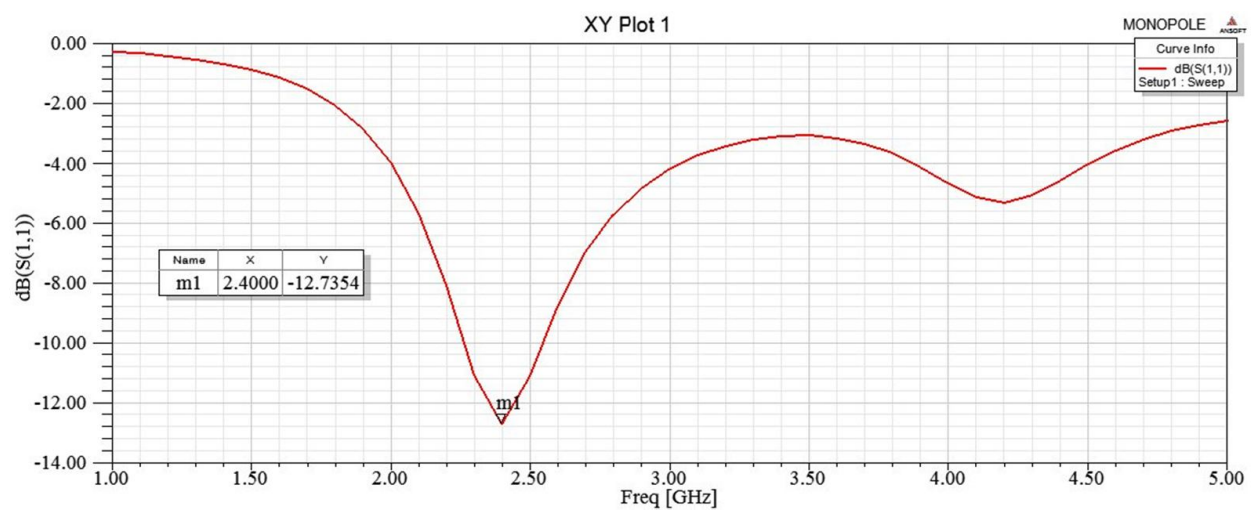

Figure 5.Simulation results of monopole antenna (Frequency verses S11)

Same antenna design is modified to design monopole antenna like $\mathrm{T}$ shape. The $\mathrm{T}$ shape monopole prototype and its simulation results in terms of frequency verses S11 is as shown in Fig. 6

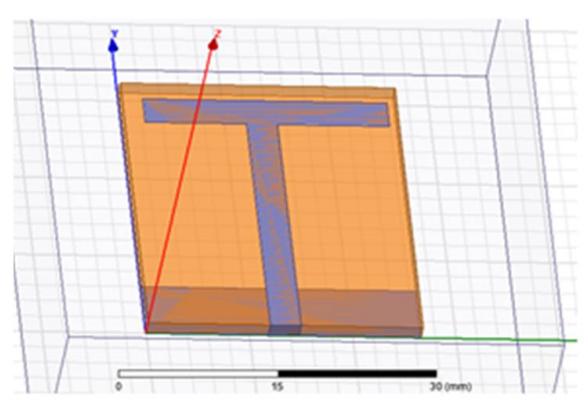

(a)

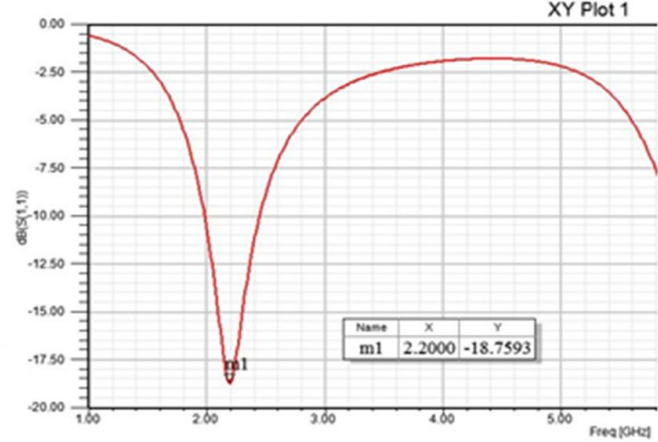

(b)

Figure 6. (a) T type monopole (b) S11 response for T type monopole 


\section{B. T shape Monopole coupled with Split Ring Resonator}

As per previous results for the $\mathrm{T}$ type monopole antenna it is tuned to $2.2 \mathrm{GHz}$. here the author wants to design multiband antenna as per reviews in various papers it is clear that multiband antenna design is possible by using the concept of Split Ring Resonator(SRR). Here rectangle SRR is used to design multiband antenna as shown in Fig. 7.

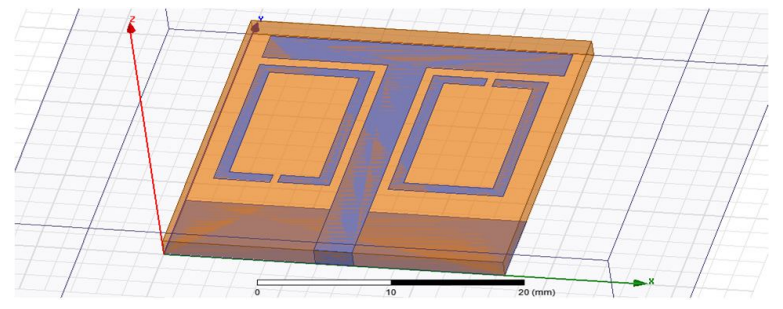

Figure $7 \mathrm{~T}$ shape antenna coupled with SRR

The antenna is simulated in HFSS and simulated. Simulated results in Fig 8. shows that when T type monopole is coupled with SRR it gives multiple bands. Desired bands can be tuned by resonating the SRR.

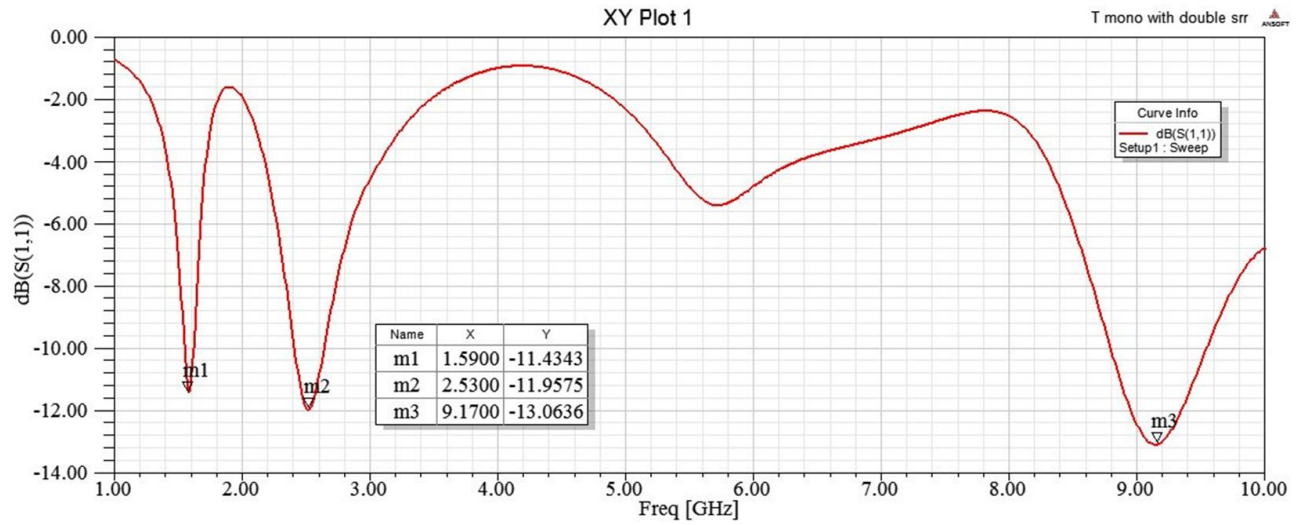

Figure.8. Return Loss verses frequency response for T shape monopole antenna coupled with SRR

The comparative results for Monopole T shape monopole and T shape monopole with SRR is as shown in Fig. 9

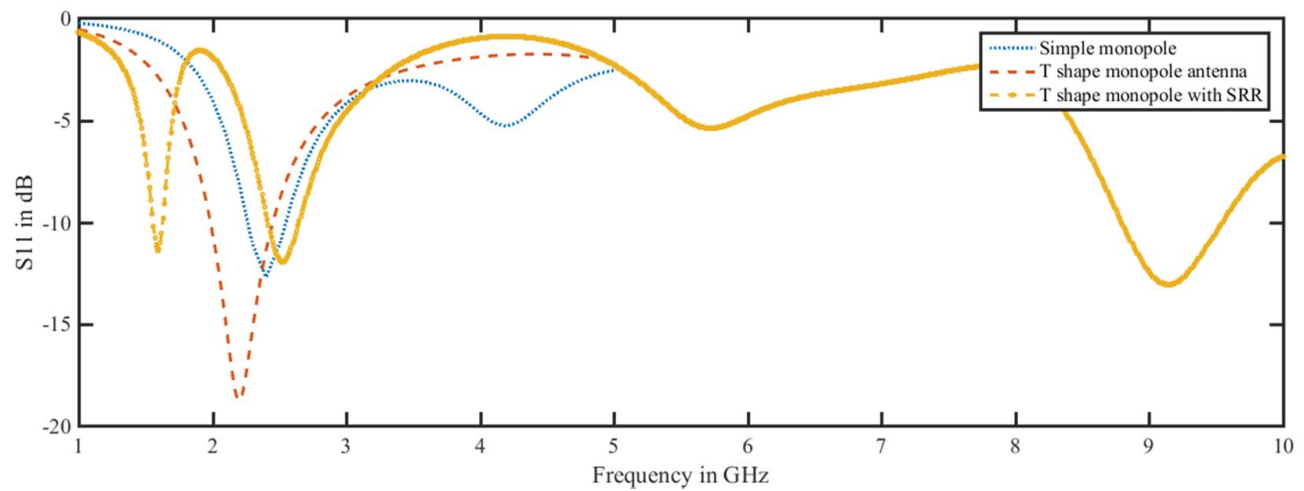

Figure 9. Comparative results for Monopole T shape monopole and $\mathrm{T}$ shape monopole with SRR

\section{Multib and Antenna using Split Ring Resonator}

Monopole antenna structure is modified using the concept of metamaterials i.e. by modifying the monopole antenna using SRR coupling. Monopole antenna is now coupled with Complementary Split Ring Resonators 
(CSRR) on the top surface as in Fig 10 (a). Ground also modified with double split ring resonators on both ends as shown in Fig 10(b). The said antenna is simulated in HFSS and is as shown in Fig 11 and its frequency response in Fig.12.

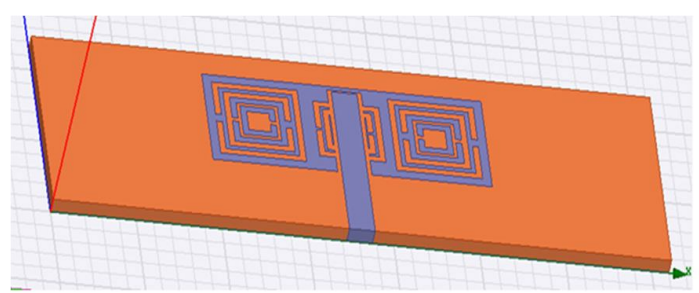

(a)

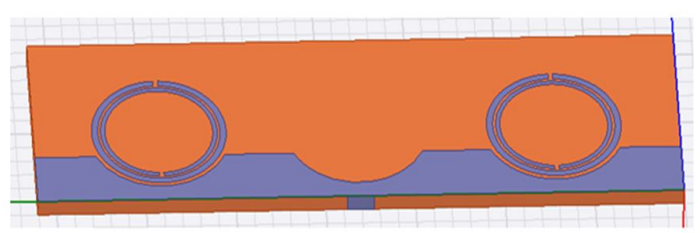

(b)

Fig. 10 Multiband antenna using SRR and CSRR a) Top view b) Ground plane

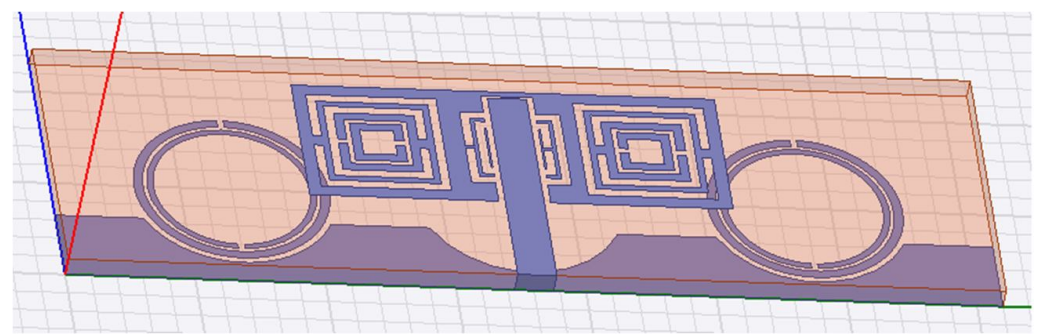

Fig 11 Multiband antenna prototype using SRR and CSRR in HFSS

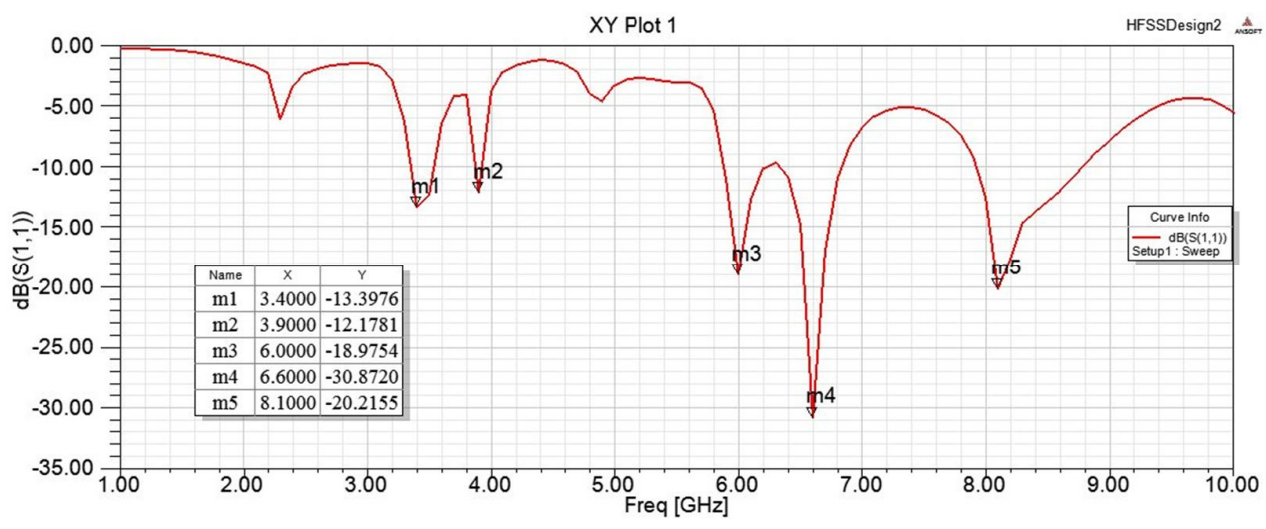

Fig. 12. S11 verses frequency response for antenna

\section{A. Effect of Split Ring Resonator on multiband Operation}

The ground structure of antenna shown in Fig. 11 is modified as shown in Fig.13.

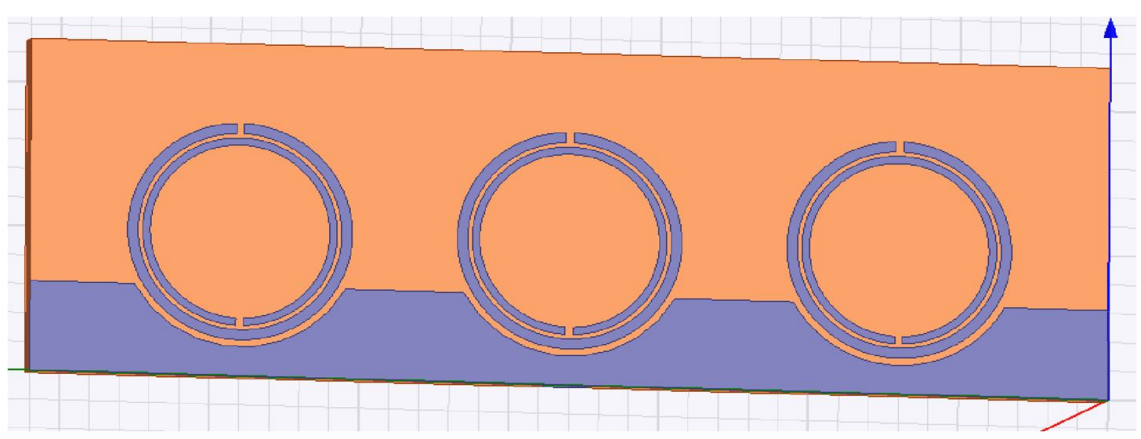

Figure.13. modified ground structure of antenna 
It is observed that the after placing SRR at the centre of ground plane the antenna behaves as that of UWB antenna in the frequency range of $6 \mathrm{GHz}$ to $9.5 \mathrm{GHz}$ range as shown in figure 14 . From the simulation results and the author concludes that Multiband to UWB antenna conversion is possible by modifying the SRR and CSRR structures.

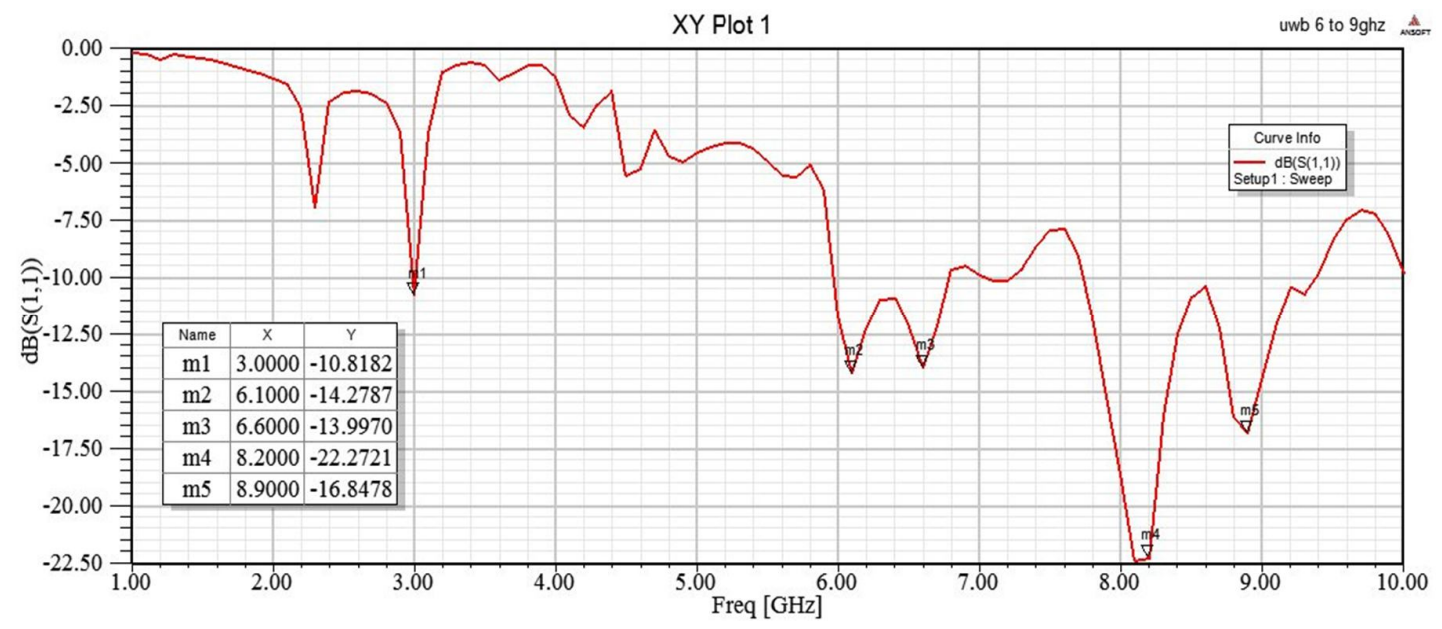

Figure 14. S11 verses frequency response after modification in ground structure

Comparative results for the antenna in with ground structure for multiband antenna and modified ground structure as shown in Fig 15.

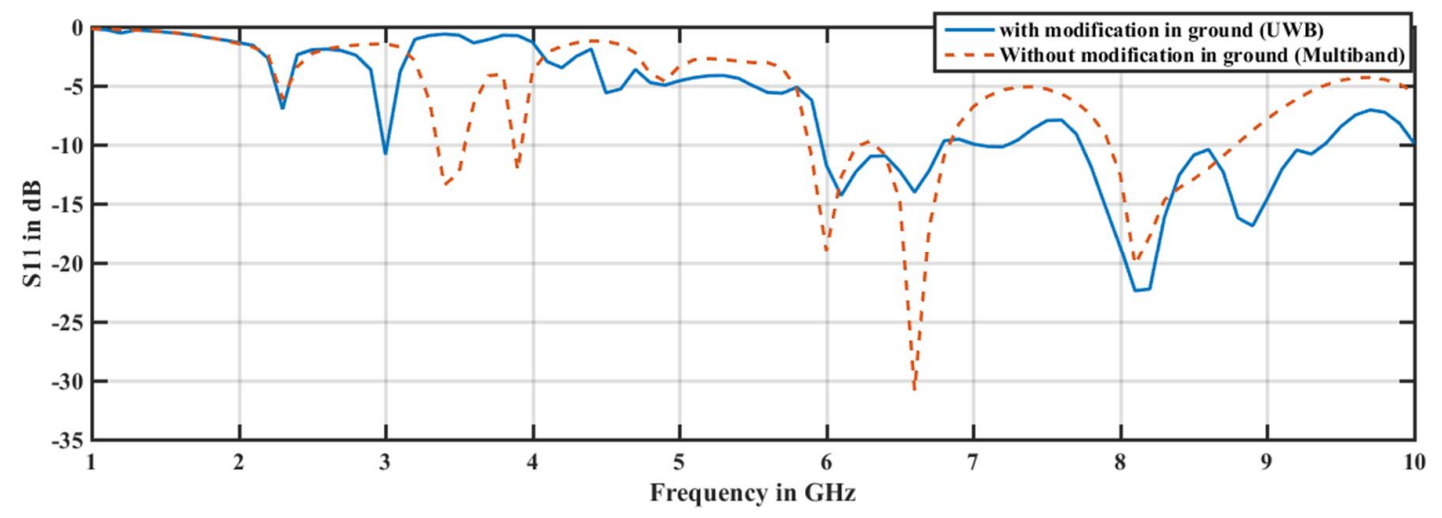

Figure 15 comparative results before and after modification of ground structure with SRR

\section{CONCLUSION}

Now days metamaterials and its application as antenna is growing field in the area of research. This paper reviews the recent development of multiband antennas using different types of metamaterial concepts. In this paper author have discussed metamaterials based on concept of SRR and CSRR. Split Ring resonators are used to design multiband antennas as well as UWB antennas of desired frequency. In this paper effect of SRR on monopole antenna is analyzed. It is observed that SRR can be used to design the multiband antenna and UWB antennas with smaller size compared with others. Presently many communication systems needs multiband antennas with smaller size and this growing need can be fulfilled with the help of this technique. Metamaterials find its applications in various fields such as satellite communications, WLAN / Mobile communications, and on-body communications. Metamaterials received great attention in research areas of physics and engineering. Microwave and millimeter wave applications of metamaterials are as couplers, resonators, negative refractive index lenses, small antennas, leaky wave antennas and absorbers. Some 
interesting application of metamaterials is cloaking and invisible metamaterials. Fabricating the same structure using the FR4 or any other substrate and testing with VNA will be future scope for this paper.

\section{REFERENCES}

[1] Christophe Caloz, Tatsuo Itoh, "Metamaterials: Transmission line Theory and Microwave Applications the Engineering Approach". A John Willy \& Sons Inc., Publication, 2006

[2] Nader Engheta, Richard W. Ziolkowski, "Metamaterials Physics and Engineering Explorations," A John Willy \& Sons ,Inc.,Publication, 2006.

[3] Marek S. Wartak, Kosmos L. Tsakmakkidis, Ortwin Hess, "Introduction to metamaterials", Physics in Canda Vol.67, No.1, Jan-Mar 2011.

[4] V. G. Veselago "The electrodynamics of substances with simultaneously negative values of $\varepsilon$ and $\mu$ "., Soviet Physics Uspekhi, Volume 10, Number 4, January-February 1968.

[5] Rajender Singh, Girish Kumar, "Broadband Planar Monopole Antennas" M.Tech credit seminar report, Electronic System group,EE Department, IIT Bombay, 2003

[6] K. P. Ray, "Design Aspects of Printed Monopole Antennas for Ultra-wide Band Applicatons", Hindawi Publishing Corporation, International Journal of Antennas and Propagation, Volume 2008, Article ID 71385.

[7] Slyusar v.i "metamaterials on antenna solutions", International Conference on Antenna Theory and Techniques", Lviv, Ukraine pp. 19-24, 6-9 October, 2009

[8] Rakhesh Singh Kshetrimayum, "Printed Monopole Antennas For Multiband Applications". International Journal Of Microwave And Optical Technology ,Vol. 3, No. 4, September 2008.

[9] Claudio G. Parazzoli, Robert B. Greegor, Minas H. Tanielian, "Applications of Metamaterials in the GHz Frequency Domain" IEEE 2007

[10] Yuandan Dong,Tatsuo Itoh "Metamaterial-Based Antennas", Proceedings of the IEEE, Vol. No. 7, July 2012.

[11] In Kwang Kim, Huan Wang, Steven J. Weiss, Vasundara V. Varadan, "Embedded Wideband Metaresonator Antenna on a High-Impedance Ground Plane for Vehicular Applications. 